\title{
Learning by doing: the value of case studies of health impact assessment
}

\author{
Ben F. Harris-Roxas ${ }^{\mathrm{A}, \mathrm{B}}$ and Patrick J. Harris ${ }^{\mathrm{A}}$ \\ ${ }^{\mathrm{A}}$ Centre for Health Equity Training, Research and Evaluation \\ (CHETRE), University of New South Wales \\ BCorresponding author.Email: b.harris-roxas@unsw.edu.au
}

\begin{abstract}
The nine health impact assessment (HIA) case studies in this issue represent a considerable contribution to the HIA literature and provide a number of lessons. These lessons include the value of using evidence in HIA to aid decisionmaking; the various forms that stakeholder and community involvement in HIA can take; and the fact that HIA can act as a catalyst for intersectoral engagement. They also highlight challenges faced by HIA practitioners, including time, methods of assessment, developing evidence summaries and considering equity.
\end{abstract}

Much of what has been written on health impact assessment (HIA) has focussed on what health professionals and impact assessors 'should do'. HIA 'should' be undertaken early enough in planning to allow changes to be made; 1 HIA 'should' be thought of as a group of research activities; HIA 'should' use a range of data; ${ }^{2}$ and HIA 'should' involve robust methods and evaluation and monitoring. ${ }^{3}$ The nine case studies included in this issue represent a significant shift from 'should do' to 'did'.

As cases based on the practice of HIA, the articles discuss the benefits of HIAs in urban settings and the impact they have on decision-making. They also challenge us to improve the ways we conduct HIA. In addition, the cases highlight the useful role that HIA can play as a tool for health systems seeking to respond to the potential health impacts of urbanisation and growth.

The case studies included in this issue of the Bulletin have been conducted on a wide variety of proposals and across a wide range of settings, including local government, neighbourhood renewal, strategic development and population growth, land use planning, social planning and capital works projects. Despite their diversity, they offer common lessons. This article summarises some of those lessons.

\section{Value of evidence to decision-making}

Many of the case studies emphasise the positive role that evidence regarding potential health impacts played in influencing and assisting decision-making, resulting in issues being considered that otherwise might not have been. The case studies also support the notion that evidence can supplement the frameworks that are currently used to develop and implement proposals, such as best practice models. ${ }^{4,5}$

\section{Community and stakeholder involvement}

Stakeholder and community involvement in the HIA can take various forms and serve several purposes. Stakeholders' views about potential impacts may be explicitly sought through the identification step ${ }^{6}$ or they may be consulted to assist with the assessment and prioritisation of impacts. Community and stakeholder comment may also be sought on the draft HIA report and its recommendations. As the case studies demonstrate, stakeholder engagement can enhance the acceptability of a HIA's recommendations and identify issues that may not have been considered in planning and decision-making.

\section{Intersectoral engagement}

HIA may act as a catalyst for intersectoral engagement. This can occur by going through the process of the HIA, as happened with the Christchurch HIA (see Stevenson et al. in this issue), or the HIA may spawn subsequent activities, as occurred with the Granville HIA (see Tennant and Newman in this issue). The case studies show that HIA can live up to the rhetoric about ensuring that potential health impacts are considered in health and other sectors' decision-making and in the development and implementation of new activities.

\section{Range of impacts considered}

The case studies also demonstrate that HIAs can successfully examine a broad range of potential heath impacts (Table 1) that include both health protection and health promotion issues.

\section{Evaluation}

Internationally, it is acknowledged that there has been a lack of evaluation of completed HIAs. ${ }^{7}$ This issue of the Bulletin represents a substantial step towards addressing this deficit. Most of the case studies in this issue include an evaluation of the process, examining how the HIA was conducted and how it was perceived by those involved. Several studies also include details of impact evaluations 
Table 1. Summary of the impacts considered in the health impact assessment case studies included in this issue of the NSW Public Health Bulletin

\begin{tabular}{ll}
\hline Air quality & Physical activity \\
Asbestos exposure & Safety and perceptions of safety \\
Buffering and exposure to toxins & Social cohesion \\
Community and social services & Street-level design and local urban form \\
Economic development and business & Suburban and regional urban form \\
Food and nutrition & Traffic \\
Housing & Transport \\
Injury & Waste management \\
Parking & Water availability and quality \\
\hline
\end{tabular}

that examined the extent to which the recommendations of the HIAs were implemented and what related changes occurred as a result of the HIAs.

\section{Challenges in conducting HIAs}

Four major challenges are evident in the HIA case studies:

- time constraints

- methods used in the assessments

- availability of synthesised and summarised evidence

- equity.

\section{Time}

Time is a recurrent issue in the case studies. Often, there is only limited time available to undertake HIAs. This is partly due to the speed with which decisions are made once a proposal is detailed enough to assess. Compounding this, many of the HIAs in this issue represent the first HIAs that were undertaken by their agencies, and the HIA practitioners had to learn 'on the job'. However, this situation may have produced benefits partner agencies engaged with the learning process and provided important insights into how decisions would be made regarding the proposal, emphasising the value of learning by doing.

\section{Identifying potential impacts}

The methods used to identify potential health impacts in the case studies include literature reviews, key informant interviews, stakeholder workshops and analysis of options and scenarios. Although these methods are appropriate, the challenge for HIA is to enhance the rigour of the methods used while still remaining timely and relevant to decision-making. This will not only improve the predictive accuracy of HIAs, but also ensure that assessments will be defensible when subjected to detailed scrutiny. This aspect will become more important as the use of HIAs becomes more widespread.

\section{Summaries of evidence}

Summaries of evidence assisted in several the case studies, enabling the assessors to reliably identify and predict potential impacts in a timely fashion. This highlights the need for consolidated summaries of the health impacts of a range of activities. These summaries are not required on issues where numerous reviews of the evidence already exist, such as the impacts on health of the built environment and urban form. ${ }^{8-10}$ Rather they are needed on important and somewhat neglected issues such as water and social cohesion, both issues that were considered in several of the HIAs in this issue.

\section{Health equity}

Considering the distribution of impacts is a feature of many of the HIA case studies, though this largely takes the form of looking at the impacts on Indigenous groups. While essential, this fails to meet the minimum criteria for considering differential impacts within HIA in terms of:

- age

- gender

- socioeconomic position

- culture and ethnicity

- locational disadvantage

- existing levels of health and disability. ${ }^{11}$

A meaningful consideration of health equity is a substantial challenge facing HIA. If HIAs fail to systematically assess the distribution of potential impacts not only will they be failing to realise one of their key principles, ${ }^{12}$ but they will also be undermining a key element of the utility and effectiveness of HIAs. For example, rates of diabetes in Australia are twice as high in the poorest areas compared with the wealthiest ones. ${ }^{13}$ If we fail to adequately assess how the impact of measures designed to curb or address diabetes will be distributed, we will undermine their effectiveness at a population level.

Addressing health equity in HIAs is therefore not simply a social justice issue but a central concern in ensuring the sustainability of urban environments, health systems and the long-term use of HIAs. The Equity Focused Health Impact Assessment Framework provides clearly structured guidance for practitioners seeking to improve the consideration of equity within their HIAs. ${ }^{14}$ 


\section{Conclusion}

These case studies reflect the growing pool of experience of HIA in action and the considerable expertise in HIA that exists in Australia and New Zealand. They provide considerable insight into how HIA works in practice, the challenges faced and the benefits that can be derived from undertaking HIAs in urban settings.

\section{References}

1. Scott-Samuel A. Health impact assessment: theory into practice. JECH 1998; 52(11): 704-5.

2. Lock K. Health impact assessment. BMJ 2000; 320: 1395-8. doi:10.1136/bmj.320.7246.1395

3. Parry J, Stevens A. Prospective health impact assessment: pitfalls, problems, and possible ways forward. BMJ 2001; 323: 1177-82. doi:10.1136/bmj.323.7322.1177

4. Menzies T. Reflections on the ways HIA can be made most useful to local government in NSW. Sydney: UNSW Research Centre for Primary Health Care and Equity, 2007.

5. Blau G, Mahoney M. The positioning of health impact assessment in local government in Victoria. Melbourne: Deakin University, 2005.

6. Simpson S, Harris E, Harris-Roxas B. Health impact assessment: an introduction to the what, why and how. Health Promot J Aust 2004; 15: 162-7.

7. Quigley R, Taylor L. Evaluating health impact assessment. Public Health 2004; 118: 544-52. doi:10.1016/j.puhe. 2003.10.012
8. Lavin T, Higgins C, Metcalfe O, Jordan A. Health impacts of the built environment: a review. Dublin: Institute of Public Health in Ireland, 2006.

9. Mead E, Dodson J, Ellway C. Urban environments \& health: identifying key relationships \& policy imperatives. Brisbane: Griffith University, 2006.

10. Gebel K, King L, Bauman A, Vita P, Gill T, Rigby A. et al. Creating healthy environments: a review of links between the physical environment, physical activity and obesity. Sydney: NSW Health Department and NSW Centre for Overweight and Obesity, 2005.

11. Harris-Roxas B, Simpson S, Harris E. Equity focused health impact assessment: a literature review. Newcastle: Australasian Collaboration for Health Equity Impact Assessment, 2004.

12. ECHP. Gothenburg consensus paper on health impact assessment: main concepts and suggested approach. Brussels: European Centre for Health Policy, WHO Regional Office for Europe, 1999.

13. Comino E, Hermiz O, Flack J, Harris E, Powell Davies G, Harris M. Using population health surveys to provide information on access to and use of quality primary health care. Aust Health Rev 2006; 30: 485-95.

14. Mahoney M, Simpson S, Harris E, Aldrich R, Stewart Williams J. Equity focused health impact assessment framework. Newcastle: Australasian Collaboration for Health Equity Impact Assessment, 2004. 